\title{
THE USE OF CONCENTRATED HUMAN SERUM ALBUMIN IN THE TREATMENT OF CIRRHOSIS OF THE LIVER ${ }^{1}$
}

\author{
BY HENRY G. KUNKEL, DANIEL H. LABBY,2 EDWARD H. AHRENS, JR., \\ ROBERT E. SHANK, ${ }^{3}$ AND CHARLES L. HOAGLAND 4 \\ (From the Hospital of The Rockefeller Institute for Medical Research, New York City)
}

(Received for publication November 12, 1947)

The formation of ascites in patients with cirrhosis of the liver probably depends on at least three factors: (1) hypoalbuminemia, (2) portal hypertension, and (3) increased amounts of circulating antidiuretic substance. The relative importance of these factors varies with the individual patients. Although the formation and disappearance of ascites do not correlate perfectly with levels of serum albumin in all patients, in the majority of cases the two findings are coincident and apparently related $(1,2)$. The use of concentrated human serum albumin in these patients for its osmotic effect would be expected to cause a diuresis. The extent to which this diuresis is produced would depend in large part upon the relative importance of the various factors of ascites production, being most marked in those patients in whom hypoalbuminemia is most specifically at fault.

The observations of Janeway (3) and Thorn (4) and their co-workers in patients with liver disease demonstrated that the albumin level of the serum could be raised to normal by repeated injections of albumin. Certain of their patients showed a loss of edema and ascites but the oncotic effects were disappointing in their experience.

\footnotetext{
1 The serum albumin used in this study was supplied in part by the Bureau of Medicine and Surgery, United States Navy, and in part by the American National Red Cross. It was prepared from blood collected by the American Red Cross from voluntary donors.

This is one of a series of investigations on serum albumin being carried out with material supplied by the American National Red Cross. As soon as sufficient data become available to justify final conclusions concerning its therapeutic value, a full report to the medical profession on the use of serum albumin in medical practice will be published.

2 Present address: University of Oregon Medical School, Portland, Oregon.

${ }^{3}$ Present address: Public Health Research Institute of The City of New York, Inc., New York.

4 Deceased, August 2, 1946.
}

While the largest portion of the administered albumin did not remain in the blood stream, nitrogen balance studies showed that this protein had been retained, presumably in the tissues. The question as to whether parenterally administered albumin is utilized in building tissue protein is not completely clear, however. The work of Whipple and co-workers $(5,6)$ in dogs has indicated that plasma protein may replace tissue protein without being broken down to its constituent amino acids. Albright and co-workers (7) by measuring phosphorus and potassium as well as nitrogen balance, have obtained evidence for the utilization of part of the injected albumin for the building of tissue protein in certain patients.

In addition to its oncotic and nutritional properties, albumin probably has other important functions in the body. It has the remarkable capacity to enter into combination with many molecules and ions in a manner different from other proteins (8). This property also has made albumin a valuable supplement to a medium for the growth of tubercle bacilli (9). The significance of the binding effect of albumin in the living organism has not been fully determined but it undoubtedly is important in the transport of various substances through the body. In view of these specific effects of albumin, it is to be expected that albumin therapy in liver disease would produce profound metabolic results.

\section{MATERIALS AND METHODS}

The present study represents the results of albumin therapy in 17 patients with severe liver disease. All but two of them demonstrated ascites at the time when albumin was first administered. The diagnosis was wellestablished by a characteristic history, numerous physical examinations, $x$-ray studies of the esophagus and abdomen, and a wide variety of liver function studies. In eight of the patients the diagnosis was confirmed by the gross appearance of the liver and from sections of the liver obtained at laparotomy or autopsy. All patients were hospitalized at the Hospital of The Rockefeller 


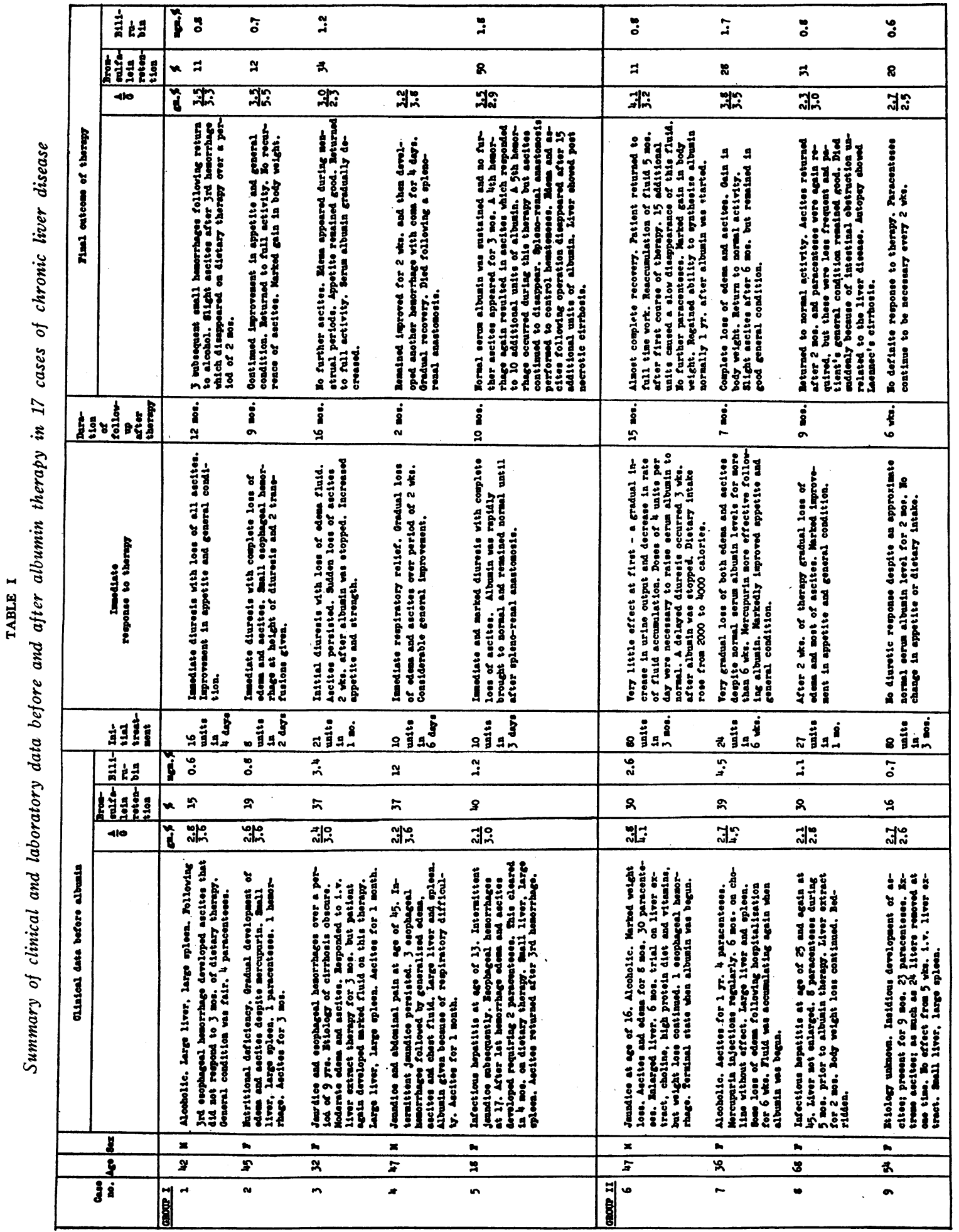




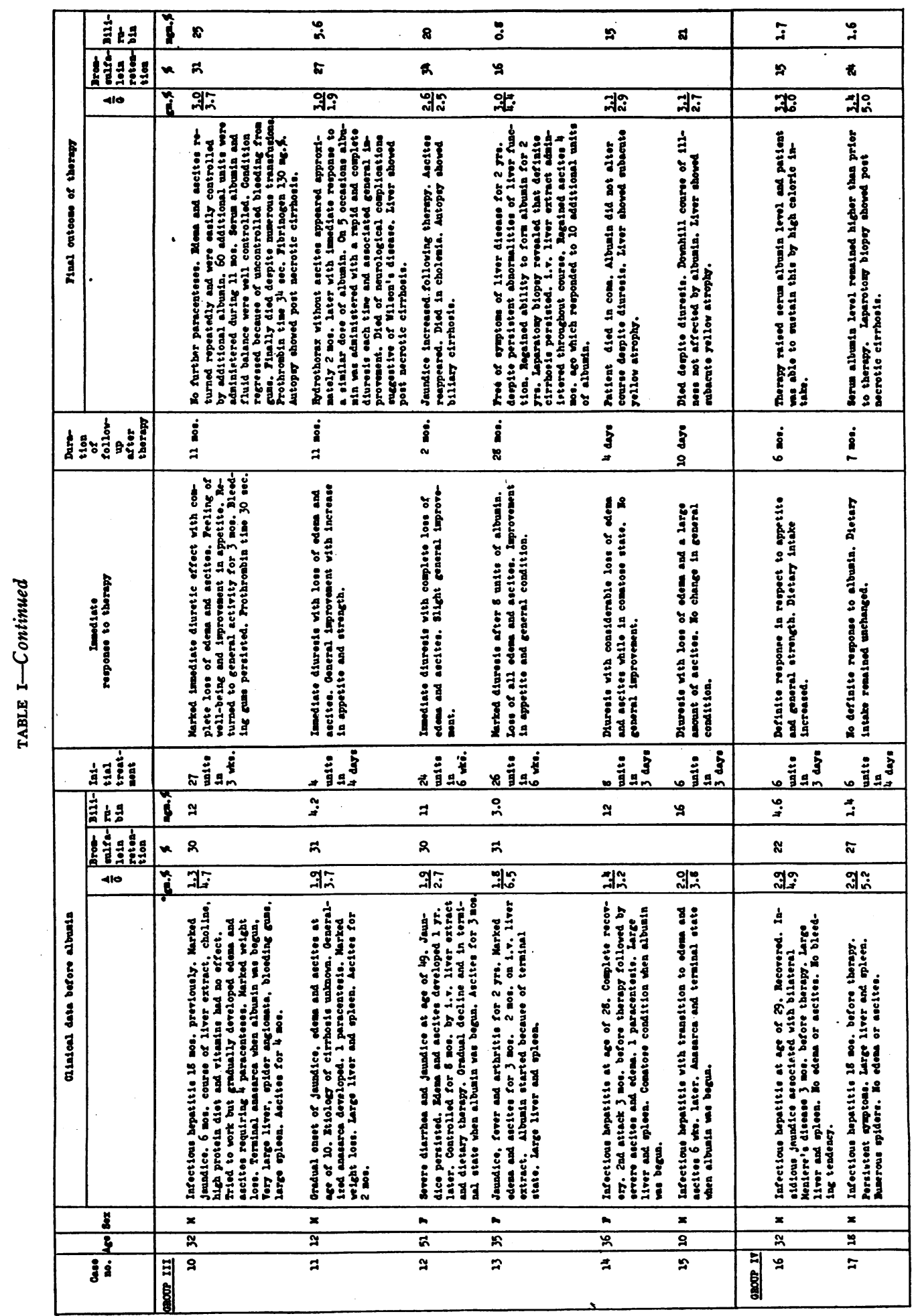


Institute during the period of albumin therapy. When sufficient improvement had been accomplished by hospitalization, the patients were observed twice weekly in the Out Patient Department.

Etiology of the 15 cases of cirrhosis in this series was varied. Four gave a clear-cut history of chronic alcoholism with associated nutritional deficiencies; four were patients with cirrhosis of the liver following infectious hepatitis; in the remaining seven the etiology of the cirrhosis was obscure.

In addition, two patients with subacute yellow atrophy following infectious hepatitis were treated with albumin. Both of these patients had edema and ascites and were in a critical state when treatment was begun; the diagnosis was confirmed at autopsy.

In studies of the changes in caloric intake during the course of albumin therapy, careful records of the daily intake of protein, fat and carbohydrate were kept by trained dietitians. An effort was made to provide patients with an excess of food and calculations of the intake were made from the amounts offered and refused. Diets were kept high in protein; fats and carbohydrates were provided according to the taste of the individual. For nitrogen balance studies three-day collections of urine and feces were analyzed for total nitrogen by the macromethod of Kjeldahl; nitrogen intake was calculated from standard tables.

Salt was not restricted in the diet. Preliminary studies indicated that with such a restriction the caloric intake fell, and, since therapy was chiefly directed toward increasing caloric intake, salt restriction was not continued.

Alterations in serum proteins under the influence of albumin were studied in detail in each case. Serum albumin and globulin were determined by the Howe method (10) with Kjeldahl digestion and nesslerization of the filtrate. Electrophoretic analyses were carried out on the serum and ascitic fluid in certain of the patients as a supplementary method of protein estimation. The effect on the serum proteins of changes in plasma volume associated with albumin therapy was carefully observed; plasma volume determinations were carried out by the method of Gibson and Evans (11).

In determining changes in liver function during the course of therapy the methods employed were identical with those presented in a previous study (12). Estimation of serum esterase activity was carried out in the Warburg manometric apparatus and the results expressed in terms of the amount of $\mathrm{CO}_{2}$ released from bicarbonate buffer following the hydrolysis of acetyl choline (13). The normal range is $40-80 \mathrm{~mm}$. $\mathrm{CO}_{2}$.

The antidiuretic activity of urines of six patients was measured by a bio-assay technic (14) in which male rats were hydrated and then injected intraperitoneally with a standard amount of dialyzed and concentrated human urine. The minutes required for the excretion of 50 per cent of the ingested water was taken as the assay time, which in normals ranges from 80-140 minutes.
Method of albumin administration. Albumin 5 was administered intravenously from a standard unit containing 25. gms. in $100 \mathrm{cc}$. of buffered diluent to which no preservative is added. This is the amount of albumin obtained from $500 \mathrm{cc}$. of plasma. The albumin used in 1945 contained $0.6-0.9 \mathrm{gms}$. of sodium per $100 \mathrm{cc}$., whereas in 1946 and 1947 the "salt-poor" product ( $0.3 \mathrm{gms}$.) was given. No marked difference between these two types in respect to diuretic effect could be ascertained.

\section{TOXIC REACTION TO ALBUMIN}

Four of the 17 patients treated with albumin developed a febrile reaction on one or more occasions approximately four hours after administration. None of these reactions was considered serious although a temperature of $104^{\circ}$ was encountered once.

Two patients (Nos. 2 and 5) developed esophageal hemorrhages during the period of albumin therapy. This occurred at a time when marked shifts in body fluids were taking place and diuresis was at its height. In neither case was the hemorrhage serious. Since patient No. 5 had bled previously on numerous occasions, it is possible that the hemorrhage was coincidental.

Cardiac complications were not encountered in any of the patients. However, another patient, not included in this series, who was given albumin for other purposes, developed pulmonary edema following 14 units of albumin from which she recovered with great difficulty.

\section{CLINICAL RESULTS}

In order to evaluate the results of albumin therapy, the 17 patients in this series have been divided into four main groups: I. Patients treated soon after the onset of ascites; II. Patients with longstanding ascites who required frequent paracenteses; III. Patients suffering from a marked albumin deficit associated with unusually severe liver damage; and IV. Patients with low serum albumin levels without edema or ascites. Table I summarizes the results of therapy in the entire series.

Group I. Five patients comprise the group of cases with early ascites. Two were the nutritional type of cirrhosis, two were cirrhosis of undeter-

\footnotetext{
5 This material was prepared at the Harvard Fractionation Laboratories, Department of Physical Chemistry, from blood collected by the American Red Cross.
} 
mined etiology, and one was cirrhosis after infectious hepatitis. Relatively small amounts of albumin produced a diuresis with loss of ascites and general improvement in each of the patients. Figure 1 illustrates the rapid loss of ascites in patient No. 5 in whom 10 units of albumin were sufficient to produce the desired diuretic effect.

Figure 2 illustrates the course of patient No. 3 who while on intravenous liver extract therapy showed some increase in the serum albumin level and some loss of the moderate edema and ascites that were present. However, the serum albumin subsequently fell and marked ascites developed. Following the administration of 9 units of albumin a diuresis occurred with loss of all edema and some ascites. Despite continued albumin therapy, further ascitic fluid accumulated and second para- centesis was necessary. After 21 units were administered, albumin administration was discontinued. Approximately two weeks later a second diuresis occurred. This patient has been followed for more than one year after the loss of ascites and has remained well. Minimal edema still develops during menstrual periods but ascites has never reappeared. The serum albumin level gradually declined following the period of therapy so that it may be assumed that a defect in the synthesis of this protein still exists but is less severe. The patient gained approximately $15 \mathrm{lbs}$. of body weight and returned to normal activity. This case illustrates the marked improvement that may occur despite persistent severe damage and impaired ability to synthesize normal amounts of albumin. The remaining three patients of the group im-

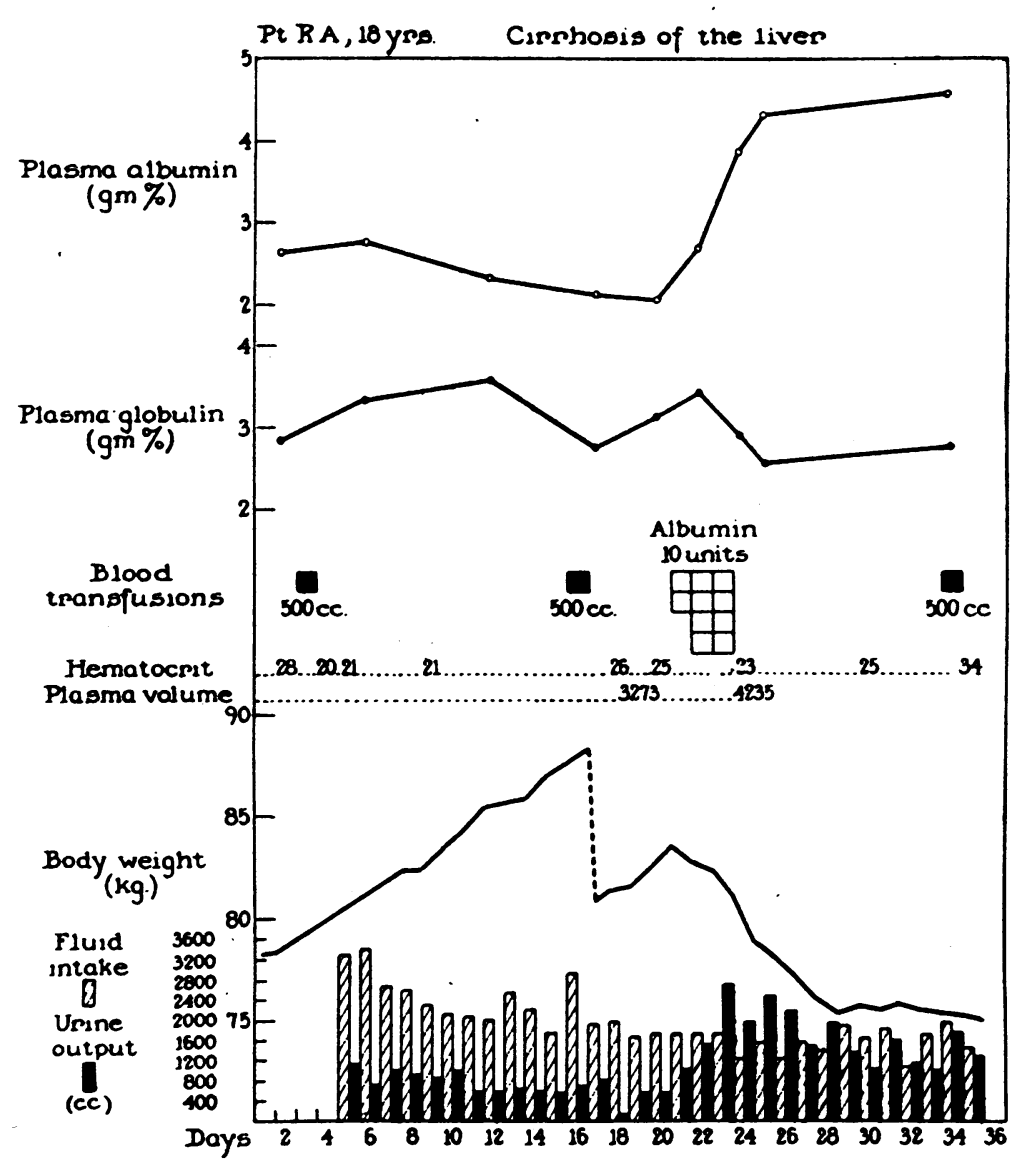

Fig. 1. Patient No. 5 (Group I) with Early Ascites Following an Esophageal Hemorreage

Rapid and complete diuresis following the administration of a small amount of albumin. 


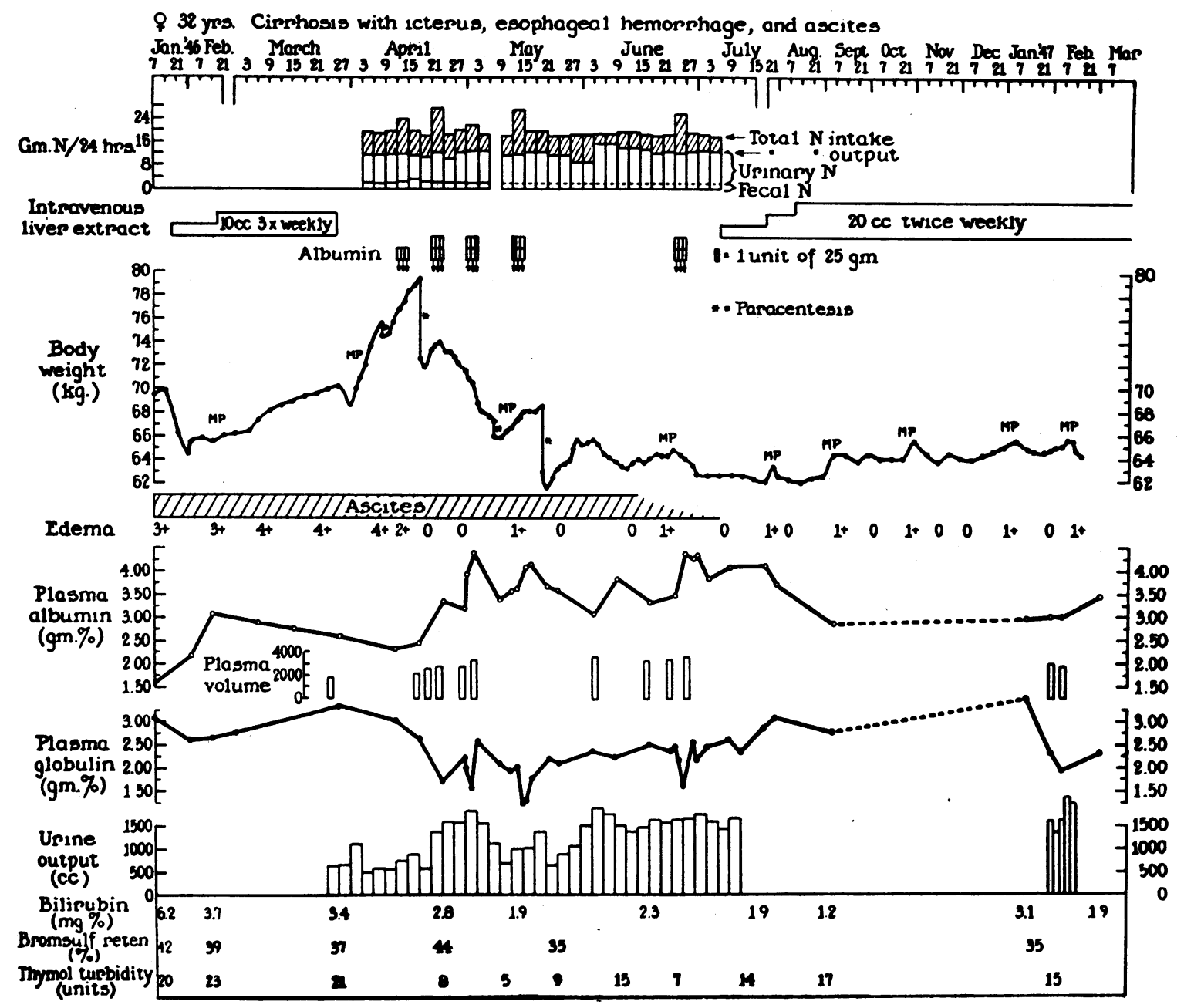

Fig. 2. Patient No. 3 (Group I) with Increasing Ascites During Liver Extract Therapy Immediate diuresis with loss of edema and delayed diuresis with loss of ascites following albumin therapy.

proved markedly on small doses of albumin. A rapid diuresis was obtained in each case. Esophageal hemorrhages have complicated the course of recovery of two of these patients, one of whom died following a spleno-renal anastomosis.

In each patient in this group albumin administration initiated a more rapid response than could have been expected with dietary and intravenous liver extract therapy. Four of the five patients were able to preserve their artificially elevated serum albumin levels by better synthesis of their own serum albumin.

Group II. This group includes four patients with a nutritional type of cirrhosis all of whom were severely ill with ascites that had been present constantly for more than five months. Each patient had been given vigorous dietary and vitamin therapy for at least three months prior to the administration of albumin. Three had been started on intravenous liver extract but, because their condition was deteriorating alarmingly, it was believed that a more rapidly acting form of therapy was necessary. This group, therefore, represents patients who would probably have succumbed to their disease despite the use of dietary and liver extract therapy.

Figure 3 illustrates the course of patient No. 6. The condition of this man grew progressively worse in the hospital while he was being given regular injections of liver extract. As may be 


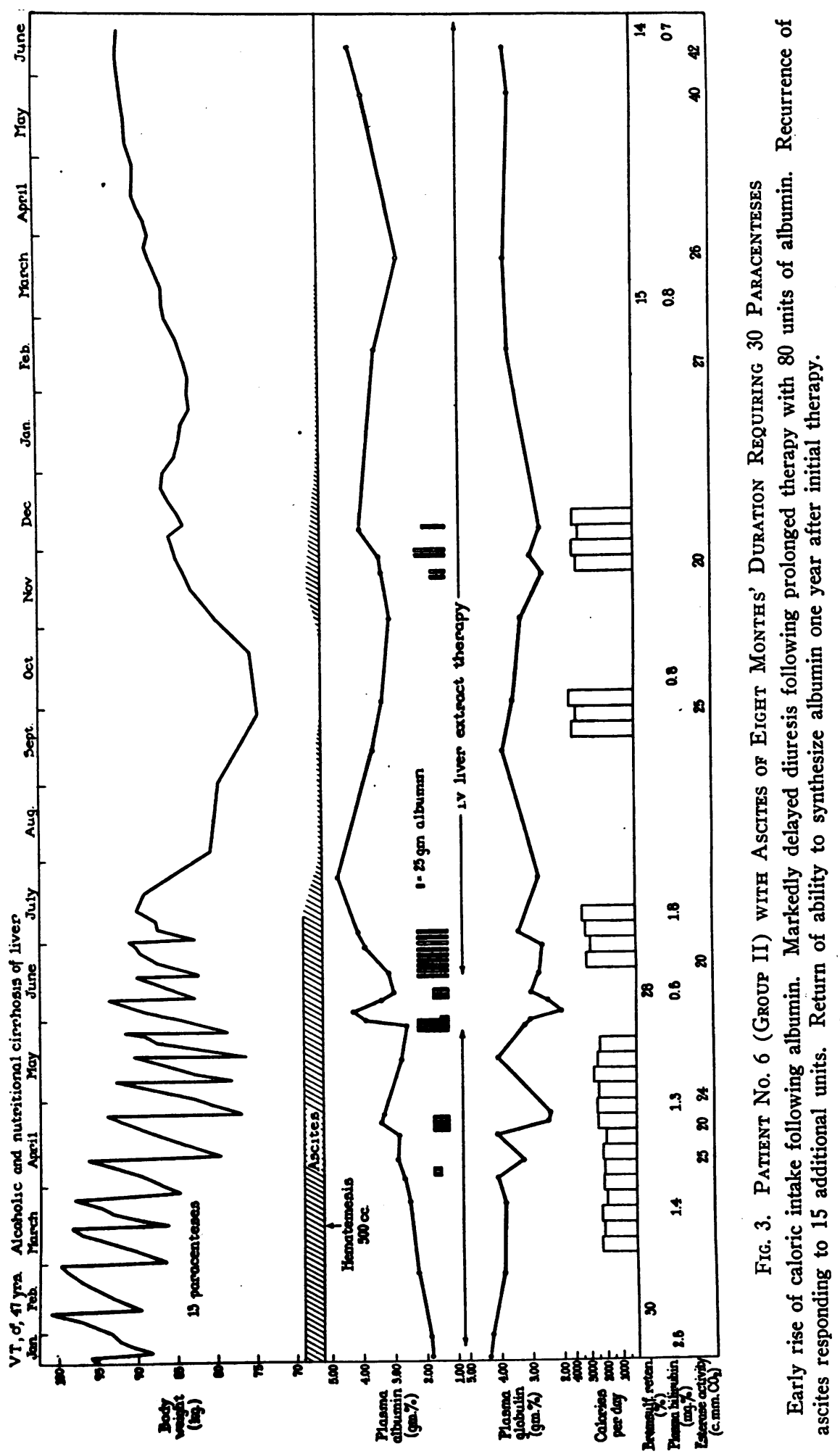


seen from the weight curve, a continuous decline in his basic weight followed each paracentesis. The administration of small amounts of albumin produced little effect. It was not until 4 units were given each day that the serum albumin level was raised to normal and a slight increase in urine volume occurred. The patient also noticed a feeling of well-being and the appearance of appetite that was reflected in an increase in caloric intake. Very little effect was noted, however, on the rate of accumulation of ascitic fluid until 4 units of albumin were administered every other day for more than two weeks. During this time a gradual slowing in the rate of fluid accumulation occurred although no acute diuresis was ever obtained. Therapy was finally stopped after 80 units of albumin had been administered despite the fact that considerable ascites persisted. Approximately two weeks after cessation of therapy a diuresis with loss of all ascites occurred. At the same time the serum albumin rose to the highest level that had been reached. Although his caloric intake was almost double the pre-treatment level, the patient failed to put on body weight. He remained free of fluid for three months but then slowly and despite continued intravenous liver extract therapy reaccumulated ascites until a paracentesis appeared necessary. Instead, 15 units of albumin were administered and a slight diuresis was obtained. Gradually over the next two months all ascitic fluid disappeared spontaneously, and the patient began to put on body weight. The improvement following the second course of albumin was more striking than after the first. The serum albumin which had gradually fallen following initial therapy was raised by the second course, but slowly fell once again. He gained approximately $30 \mathrm{lbs}$. of weight and returned to normal activity. Suddenly, six months after the last albumin treatment, the serum albumin rose spontaneously to normal indicating that the patient had finally regained the ability to synthesize normal amounts of serum albumin. His bromsulfalein retention fell from 30 per cent to 12 per cent over the period of 18 months that he was treated.

The three other patients in the group were treated with large amounts of albumin after failing to improve on other forms of therapy. All but one patient showed a gradual disappearance of ascites and marked clinical improvement. Ascites disap- peared very slowly, usually several weeks after the patients had begun to feel improved and were eating better. In patient No. 8 moderate ascites recurred three months after therapy, although the patient continued to lead a normal life. Further albumin was not administered. This patient died suddenly because of an intestinal obstruction unrelated to the liver disease. Patient No. 9, who did not respond to albumin therapy, was unusual in that she had been accumulating approximately 20 liters of ascitic fluid every 14 days for nine months prior to albumin administration. The antidiuretic titer was found to be unusually high, approximately twice the normal value (Table III). Eighty units of albumin were administered intravenously over a period of three months without noticeable effect. Although the serum albumin level was kept above 3.5 gms. per cent during this period, paracenteses continued to be necessary every 10 to 16 days.

Group III. Four patients with extremely severe cirrhosis and two patients with fatal subacute infectious hepatitis, all showing albumin levels below 2 gms. per cent, comprise this group. All demonstrated large amounts of edema associated with ascites. Laparotomy biopsy was performed on one patient, and autopsies were obtained on the five patients who died. None of the patients showed a typical Laennec's cirrhosis. The diagnosis was post-necrotic cirrhosis in three patients, biliary cirrhosis in one, and subacute yellow atrophy in two.

All patients demonstrated a diuretic response with disappearance of edema and ascites to an average of 16 units of albumin. Figure 4 illustrates the course of a typical case in this group. The patient (No. 10) was a 32 year old sailor who had had a typical attack of infectious hepatitis 18 months prior to therapy. Following a severe relapse of infectious hepatitis, symptoms and signs of liver damage persisted and he was followed for one year at the Hospital of The Rockefeller Institute. Following discharge from the Navy he attempted full time work, but his symptoms became more severe and he was readmitted to the Rockefeller Hospital with generalized anasarca and ascites. His condition was critical and during a month of dietary and vitamin therapy he required three paracenteses. Following the administration of 15 units of albumin a diuresis with loss 


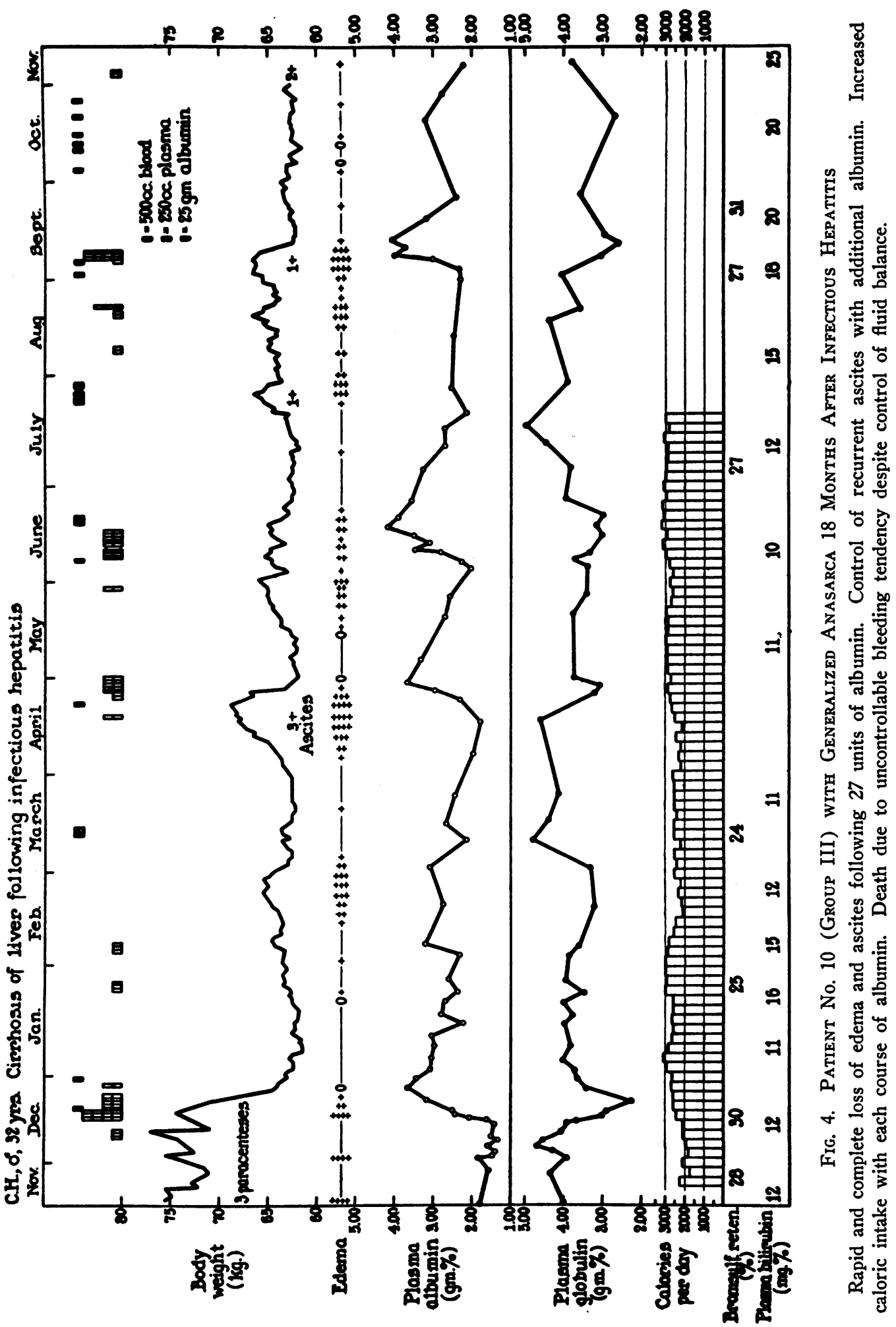


of all ascites and edema occurred, and during this diuresis 12 additional units were administered. The serum albumin level was raised to normal and the general condition of the patient improved dramatically. His caloric intake increased from 2,000 to 3,000 calories per day and within two weeks he was able to return to full activity. It was evident that he suffered from a severe albumin deficit which when overcome resulted in marked improvement. Over a period of four months following the first course of therapy the serum albumin level gradually declined and the patient once again developed ascites and edema. The administration of 12 units of albumin produced another immediate diuresis. The patient lived for approximately one year after the onset of albumin therapy, during which time recurring edema and ascites were readily controlled by albumin administration on four occasions. Albumin, however, had no measurable effect in improving his liver function, and the serum bilirubin rose in the terminal four months from $12 \mathrm{mg}$. per cent to $25 \mathrm{mg}$. per cent. Synthesis of prothrombin and fibrinogen by the liver were markedly impaired, similar in degree to the albumin deficiency. However, these proteins were not available for replacement therapy, and the patient finally succumbed because of severe, uncontrollable bleeding from his gums and nose despite numerous transfusions with fresh blood. It was believed, however, that the patient's life had been lengthened considerably by albumin therapy.

Two other patients in the group (Nos. 11 and 12) appeared to have had their life span increased by albumin administration. Patient No. 11 received five separate courses of small amounts of albumin with loss of edema and ascites on each occasion. He eventually died during curare treatment for a neurological disorder resembling Wilson's disease. Patient No. 13, who also presented a terminal picture of generalized anasarca with a very low serum albumin, demonstrated a cirrhosis of undetermined etiology associated with severe arthritis. All edema and ascites disappeared rapidly following the use of intravenous albumin. This patient differed from the others in that she continued to preserve the normal serum albumin level brought about by albumin administration and has remained well leading a normal life for 25 months following therapy. Laparotomy biopsy two years after therapy showed an advanced cirrhosis characterized by dense fibrous tissue strands and large masses of regenerating liver cells. The gratifying response to albumin in this patient emphasizes the difficulty in predicting the therapeutic effect of albumin.

The two patients with subacute yellow atrophy (Nos. 14 and 15) showed a diuresis with loss of edema and ascites while in a semi-comatose state. Their downhill course was unaffected by the albumin therapy and both died. They are additional examples of cases that react readily with a diuretic response to relatively small amounts of albumin.

The factor of portal obstruction was not apparent clinically in this group of patients with severe liver disease. Water retention corresponded closely to the albumin deficit and could be controlled readily by albumin therapy. Although three of the four patients with cirrhosis eventually died, they were markedly improved temporarily by this form of therapy. This effect, together with the dramatic and prolonged improvement in patient No. 13, demonstrates the value of albumin in this type of liver disease.

Group IV. Two patients with post-hepatitis cirrhosis without edema and ascites were given albumin therapy in order to increase dietary intake prior to the expected onset of hepatic decompensation. Patient No. 16 showed a definite increase in caloric intake and was able to maintain his artificially raised albumin level. He gained weight and was able to return to normal activity. Patient No. 17 showed no response. The dietary intake remained poor, marked fatigue persisted, and weight loss continued. However, for seven months he maintained a serum albumin level higher than that prior to therapy.

\section{SPECIFIC EFFECTS OF ALBUMIN THERAPY}

The influence of injected albumin on the ability of the liver to resume normal synthesis of albumin is perhaps the most important consideration in evaluating its therapeutic effectiveness. The necessity for periodic injections of albumin in the form of maintenance therapy rests with this question. Table II shows that four of 12 patients were able to form sufficient albumin to maintain an approximately normal level three months after the first course of therapy ended. One other patient (No. 6) regained this capacity following a second 
TABLE II

Alterations in plasma albumin level following albumin therapy in 15 patients with cirrhosis of the liver

\begin{tabular}{c|c|c|c}
\hline \multicolumn{1}{c|}{ Albumin level } & \multicolumn{3}{|c}{ No. of patients } \\
\cline { 2 - 4 } & $\begin{array}{c}\text { Before } \\
\text { therapy }\end{array}$ & $\begin{array}{c}\text { Imme- } \\
\text { diately } \\
\text { after } \\
\text { therapy }\end{array}$ & $\begin{array}{c}3 \text { mos. } \\
\text { after } \\
\text { therapy*- }\end{array}$ \\
\hline $\begin{array}{l}\text { 3.5 gms. per cent or higher } \\
\text { 3-3.5 gms. per cent } \\
3 \text { gms. per cent or lower }\end{array}$ & 0 & 15 & 4 \\
0 & 15 & 0 & 3 \\
\hline
\end{tabular}

* Three patients are not included: two have not been followed for three months following the discontinuation of therapy and one died two months after therapy.

small course of therapy. Three patients experienced a rapid fall in serum albumin after artificial elevation of this level by a series of injections and demonstrated no improvement in their ability to synthesize albumin. The remainder maintained higher levels after treatment but did not show complete restoration to normal. It should be mentioned that the patients who developed low serum albumin levels and ascites several weeks after bleeding episodes were best able to maintain permanently normal levels following therapy.
Nitrogen balance studies were carried out in four of the patients who received albumin. Figure 5 illustrates the results in a typical case. This patient was in slight negative nitrogen balance prior to therapy but then entered marked positive balance during the period of albumin administration. In the four patients studied there was greater than 90 per cent retention of injected albumin nitrogen; they lost ascites following albumin therapy and did not require further paracenteses. As a result, the continued loss of albumin through the peritoneal cavity was obviated. This was not true of the patients who required further paracenteses; they continued to lose albumin nitrogen. Further observations on the patient illustrated in Figure 5 showed that the patient remained in positive balance for at least one month after therapy was discontinued. Figure 5 also illustrates the marked difference between the effect of albumin on the nitrogen balance and of an equivalent amount of nitrogen in the form of casein hydrolysate.

A number of patients volunteered the information that they developed a feeling of well-being

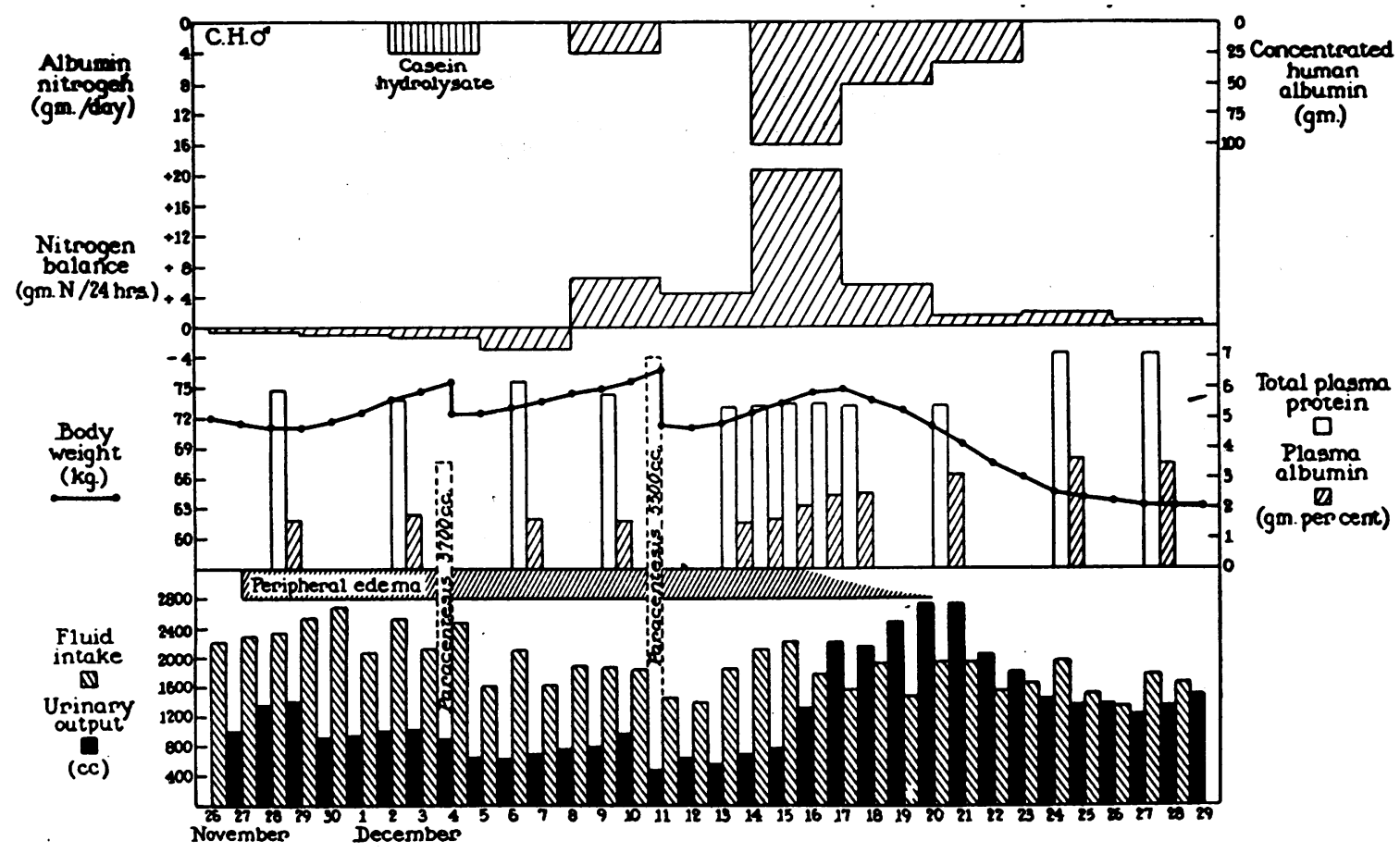

Fig. 5. Patient No. 10 (Group III). Persistent Negative Nitrogen Balance with Casein Hydrolysate

Positive nitrogen balance with an equivalent amount of albumin nitrogen. Complete retention of albumin nitrogen. 


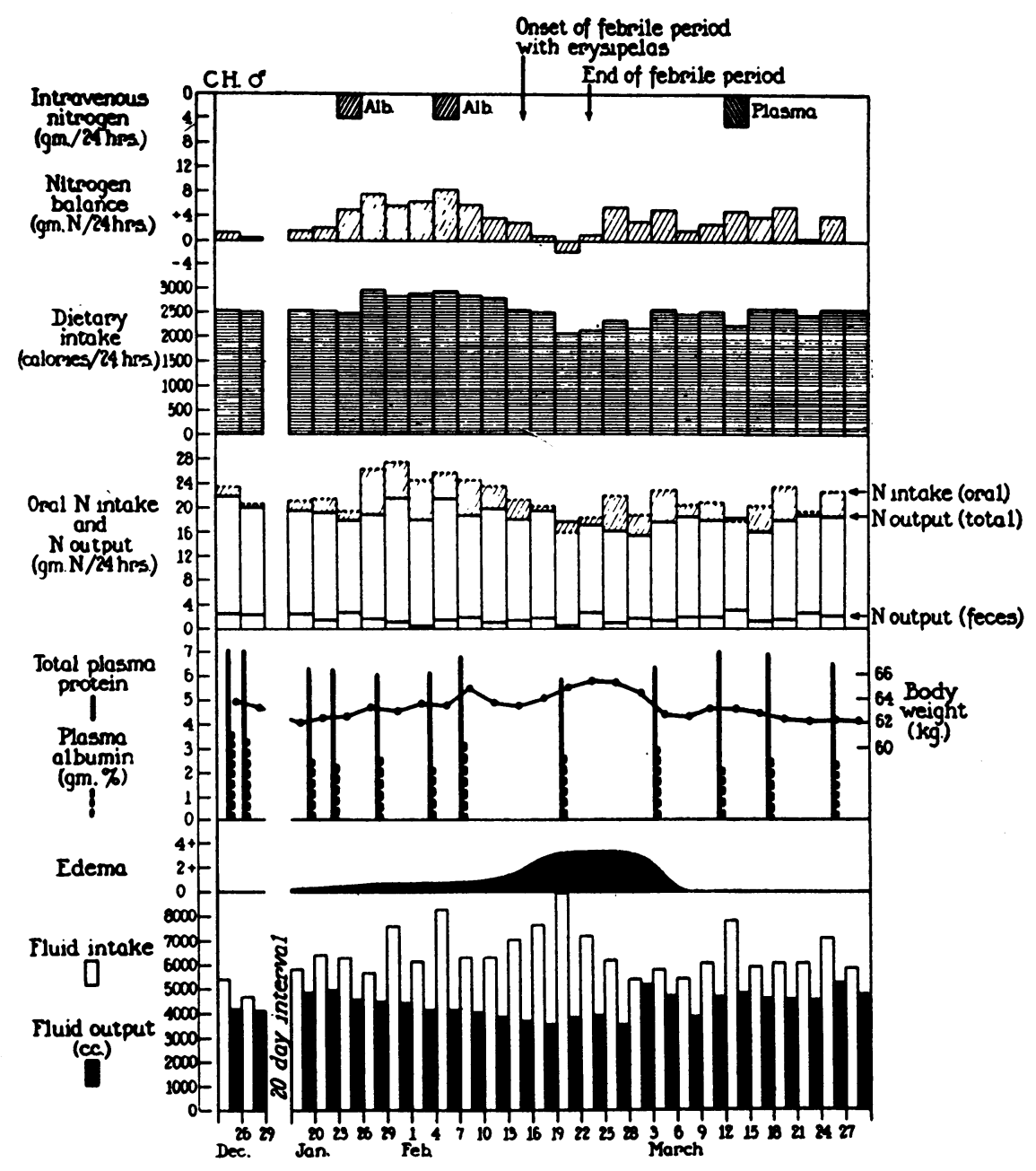

Fig. 6. Patient No. 10 (Group III). Rise in Caloric Intake and InCreased Retention of Dietary Nitrogen Following Two Courses of 3 Units of Albumin

following the administration of albumin, despite the fact that no diuresis occurred. This was associated with a general increase in appetite. To test the validity of these observations careful measurements were carried out on the caloric intake before and after albumin therapy in a patient who was free of ascites and showed only minimal edema. Figure 6 illustrates the definite rise in protein and total oral caloric intake following the administration of 3 units of albumin. Nitrogen balance determinations revealed an increased retention of dietary nitrogen in addition to the retention of the nitrogen of the albumin administered. The experiment was interrupted by a short febrile period due to a skin infection. Sub- sequent administration of plasma did not affect the caloric intake or the nitrogen balance to a similar degree. This specific effect of albumin on the dietary intake was observed during four different periods of albumin therapy in the patient (Figure 4). In another patient (Figure 3) a similar rise in caloric intake during albumin therapy was observed considerably in advance of his diuresis.

Estimation of the degree of retention of bromsulfalein was carried out before and after albumin treatment in each of the patients. No improvement in this liver function test could be demonstrated within the first month after albumin therapy. However, in four of the patients who were 
followed for more than six months following therapy, definite improvement occurred. In patient No. 6 who exhibited no jaundice the bromsulfalein retention fell from 30 per cent to 12 per cent over a period of 16 months. No immediate improvement occurred in serum bilirubin, prothrombin time, total globulin, the thymol turbidity reaction, fibrinogen, and esterase activity following albumin therapy. However, with prolonged treatment for more than six months, changes occurred in these serum components which were in general parallel to the improvement in bromsulfalein retention.

\section{DISCUSSION}

The most apparent effect of albumin in patients with liver disease was that due to its osmotic properties. In nine of 15 patients with fluid retention who were treated, a rapid loss of edema and ascites occurred. Five others showed a delayed loss of ascites. In those patients whose diuretic response to albumin was immediate, a beneficial course of events was rapidly initiated; loss of protein into ascitic fluid ceased, dietary protein intake increased, and positive nitrogen balance resulted. Such treatment carried seriously ill patients through the early critical period of their disease until such time as dietary and liver extract therapy had an opportunity to take effect.

The amount of albumin needed to produce a loss of ascites was extremely variable, ranging from 4 to 80 units, and appeared to depend upon at least four factors. The first of these was the relative role of portal obstruction in the causation of fluid accumulation. Since there is no direct method of measuring portal pressure without laparotomy, it was necessary to evaluate this from clinical signs. Esophageal varices and other evidences of collateral circulation, a small hard liver and a large spleen were considered to be associated with portal hypertension. However; the most useful sign was the amount of edema relative to the amount of ascites. Those patients exhibiting marked ascites with little or no edema were considered to have a high degree of portal obstruction, while large amounts of edema in the presence of less impressive quantities of slowly forming ascites indicated that portal obstruction, if present, was of minor importance. Edematous patients with slight evidence of portal obstruction re- sponded more readily to therapy with albumin than did patients with severe portal hypertension. Patients with subacute yellow atrophy and cirrhosis following infectious hepatitis usually fell into this category. The plasma albumin levels of this group were extremely low, occasionally below 1.5 gms. per cent, suggesting that these patients suffered from a specific albumin deficit. Approximately 12 units, administered over a period of four days, usually produced a dramatic diuresis with loss of edema and ascites. On the other hand, patients with large amounts of ascites in the absence of generalized edema, in whom portal obstruction was considered to be a dominant factor, were generally very resistant to therapy. As much as 80 units of albumin were sometimes necessary before ascites disappeared. It should be emphasized, however, that a diuresis was produced in three out of four such patients with marked evidence of portal obstruction when the plasma albumin was kept at a level of 3.5 gms. per cent or higher for a sufficiently long period of time; the one refractory patient failed to respond to 80 units given over a three-month period despite normal serum albumin levels.

A second factor of importance in determining the amount of albumin required to produce a disappearance of ascites was the rapidity with which the patient accumulated fluid. Following the administration of albumin an equilibrium between blood and ascitic fluid albumin was established, and the $A / G$ ratio in these two body compartments tended to remain equal. As a result, with the rise in albumin concentration of the serum, there was a rise in the concentration of albumin in the ascitic fluid. When the ascitic fluid collection was large and constantly reaccumulating, a sizeable drain on the injected albumin would occur with each paracentesis. Small doses of albumin were ineffective under such circumstances.

The third factor and one which has not been clearly evaluated was the length of time that fluid accumulation had been present prior to albumin therapy. Those patients treated soon after they began to accumulate ascitic fluid responded readily to therapy in each case. The patients most resistant to the diuretic effect of albumin were those who had been accumulating fluid for long periods. The experience gained by treatment of the patients in Group I suggests that early as- 


\section{TABLE III}

Comparison between the titer of antidiuretic substance in the urine and the amount of albumin required and the type of response produced by albumin therapy in six patients with cirrhosis of the liver

\begin{tabular}{c|c|c|l}
\hline $\begin{array}{c}\text { Case } \\
\text { no. }\end{array}$ & $\begin{array}{c}\text { Antidiuretic } \\
\text { titer }\end{array}$ & $\begin{array}{c}\text { Units of } \\
\text { albumin } \\
\text { required }\end{array}$ & \multicolumn{1}{|c}{$\begin{array}{c}\text { Type of } \\
\text { diuresis }\end{array}$} \\
\hline & $\begin{array}{c}\text { minutes } \\
140^{*}\end{array}$ & 10 & Immediate \\
5 & $140^{*}$ & 8 & Immediate \\
2 & $120^{*}$ & 4 & Immediate \\
11 & 300 & 24 & Very gradual \\
7 & 240 & 80 & None \\
9 & 160 & 80 & Very gradual \\
6 & & & \\
\hline
\end{tabular}

* Normal.

cites is due primarily to low serum albumin levels and that albumin therapy is a specific remedy at this time. In the patients of Group II with long-standing ascites, changes secondary to fluid accumulation have been added to the initial factor of hypoalbuminemia, rendering albumin treatment less effective. Investigations are now in progress regarding the role of alterations of peritoneal absorption in these patients.

The fourth factor influencing the amount of albumin necessary to produce a diuretic effect was the concentration of antidiuretic principle present in the urine of patients being treated. Preliminary observations indicated that those patients excreting large quantities of this substance required prolonged administration of albumin (Table III).

The ultimate effects of albumin therapy are certainly not all directly attributable to albumin alone. In certain patients this material simply initiated a curative process that was then sustained by increased caloric intake and liver extract therapy. A rise in caloric intake and increased retention of dietary nitrogen were demonstrated following albumin therapy in certain patients where the response could not be attributed to relief from the disabling effects of fluid. This effect of albumin was perhaps of even greater significance than its oncotic effects in initiating the process of recovery. In the three patients of Group II who eventually lost their ascites after prolonged albumin therapy, the effects of albumin on food intake and utilization may have been primarily responsible for the eventual disappearance of ascites. The fact that these patients demonstrated a delayed diuretic effect after albumin therapy raises the question whether supplemental nutritional and liver extract therapy which the patients were receiving continually might have been primarily responsible for improvement. However, against this possibility the following observations may be cited. First, during control periods on supplemental treatment alone these patients had failed to improve. Secondly, increased appetite and a general feeling of well-being were closely associated in time with albumin therapy. Finally, the most conclusive evidence for the primary role of albumin was obtained in those patients (Nos. 6 and 13) who developed a recurrence of ascites several months after the first period of therapy and in whom another small course of albumin caused improvement and loss of ascites for the second time.

\section{SUMMARY}

1. The results of serum albumin therapy in $\mathbf{1 7}$ patients with severe liver disease are presented.

2. Fourteen out of 15 patients with ascites lost their fluid following therapy.

3. The amount of albumin necessary to produce such an effect was variable ranging from 4 to 80 units.

4. Patients with marked evidence of portal obstruction, a high antidiuretic titer in the urine or long-standing ascites proved very resistant to therapy.

5. Patients with particularly severe liver disease and very low plasma albumin levels following infectious hepatitis responded most readily to therapy. Cases of alcoholic and nutritional cirrhosis with a short period of ascites also responded to relatively small doses of albumin.

6. Permanently beneficial results were obtained in six of seven patients with the nutritional type of cirrhosis. Two of these patients required a second short course of therapy. Patients with post-necrotic cirrhosis after infectious hepatitis and biliary cirrhosis showed only a temporary response and four of these patients eventually died.

7. Evidence was presented for certain specific effects of serum albumin on dietary intake and nitrogen balance which may be of greater importance in the results obtained than its osmotic properties. 


\section{BIBLIOGRAPHY}

1. Post, J., and Patek, A. J., Jr., Serum proteins in cirrhosis of the liver. I. Relation to prognosis and to formation of ascites. Arch. Int. Med., 1942, $69,67$.

2. Labby, D. H., Shank, R. E., Kunkel, H. G., and Hoagland, C. L., Intravenous therapy of cirrhosis of the liver. J. A. M. A., 1947, 133, 1181.

3. Janeway, C. A., Gibson, S. T., Woodruff, L. M., Heyl, J. T., Bailey, O. T., and Newhouser, L. R., Chemical, clinical, and immunological studies on the products of human plasma fractionation. VII. Concentrated human serum albumin. J. Clin. Invest., 1944, 23, 465.

4. Thorn, G. W., Armstrong, S. H., Jr., and Davenport, V. D., Chemical, clinical, and immunological studies on the products of human plasma fractionation. XXXI. The use of salt-poor concentrated human serum albumin solution in the treatment of hepatic cirrhosis. J. Clin. Invest., 1946, 25, 304.

5. Holman, R. L., Mahoney, E. B., and Whipple, G. H., Blood plasma protein given by vein utilized in body metabolism; dynamic equilibrium between plasma and tissue proteins. J. Exper. Med., 1934, 59, 269.

6. Whipple, G. H., and Madden, S. C., Hemoglobin, plasma protein and cell protein-their interchange and construction in emergencies. Medicine, 1944, 23, 215 .
7. Albright, F., Forbes, A. P., and Reifenstein, E. C., The fate of plasma protein administered intravenously. Tr. Assoc. Amer. Phys., 1946, 59, 221.

8. Bennhold, H., Uber die Vehikelfunktion der Serumeiweisskörper. Ergebn. d. inn. Med. u. Kinderh., 1932, 42, 273.

9. Dubos, R. J., and Davis, B. D., Factors affecting the growth of tubercle bacilli in liquid media. J. Exper. Med., 1946, 83, 409.

10. Howe, P. E., The use of sodium sulfate as the globulin precipitant in the determination of proteins in blood. J. Biol. Chem., 1921, 49, 93.

11. Gibson, J. G., 2nd, and Evans, W. A., Jr., Clinical studies of the blood volume. I. Clinical application of a method employing the azo dye "Evans blue" and the spectrophotometer. J. Clin. Invest., 1937, 16, 301.

12. Hoagland, C. L., and Shank, R. E., Infectious hepatitis: A review of 200 cases. J. A. M. A., 1946, $130,615$.

13. Kunkel, H. G., and Ward, S. M., Plasma esterase activity in patients with liver disease and with the nephrotic syndrome. J. Exper. Med., 1947, 86, 325.

14. Ham, G. C., Reproducible diuresis and chloruresis for bioassay of antidiuretic activity. Proc. Soc. Exper. Biol. \& Med., 1943, 53, 210. 